\section{Primary Ewing's sarcoma presenting as a Pancoast tumour}

\section{CASE REPORT}

A 48-year-old woman without a significant medical history was referred to our department after the neurologist established Pancoast's syndrome and a well-demarcated homogenous mass in the apex of the right hemithorax on MRI study of the brachial plexus. The tumour was invading the thoracic wall, adjacent musculature and the right plexus brachialis and extended into the neuroforamen of C7-T1 (figure 1B, D). She had no respiratory complaints. She had quit smoking 25 years ago and had a smoking history of only five cigarettes daily for 4 years. A PETCT scan with 18F-fluorodeoxyglucose (FDG) showed slightly increased FDG uptake (maximal standardised uptake value 4.9) without evidence of metastatic disease. The CT images showed evident destruction of the first rib on the right side (figure 1C). Histological examination of a CT-guided transthoracic needle biopsy revealed a monotonous population of small blue round cells with diffuse membranous staining for CD99 (figure 2). After demonstration of a translocation for the EWS gene by
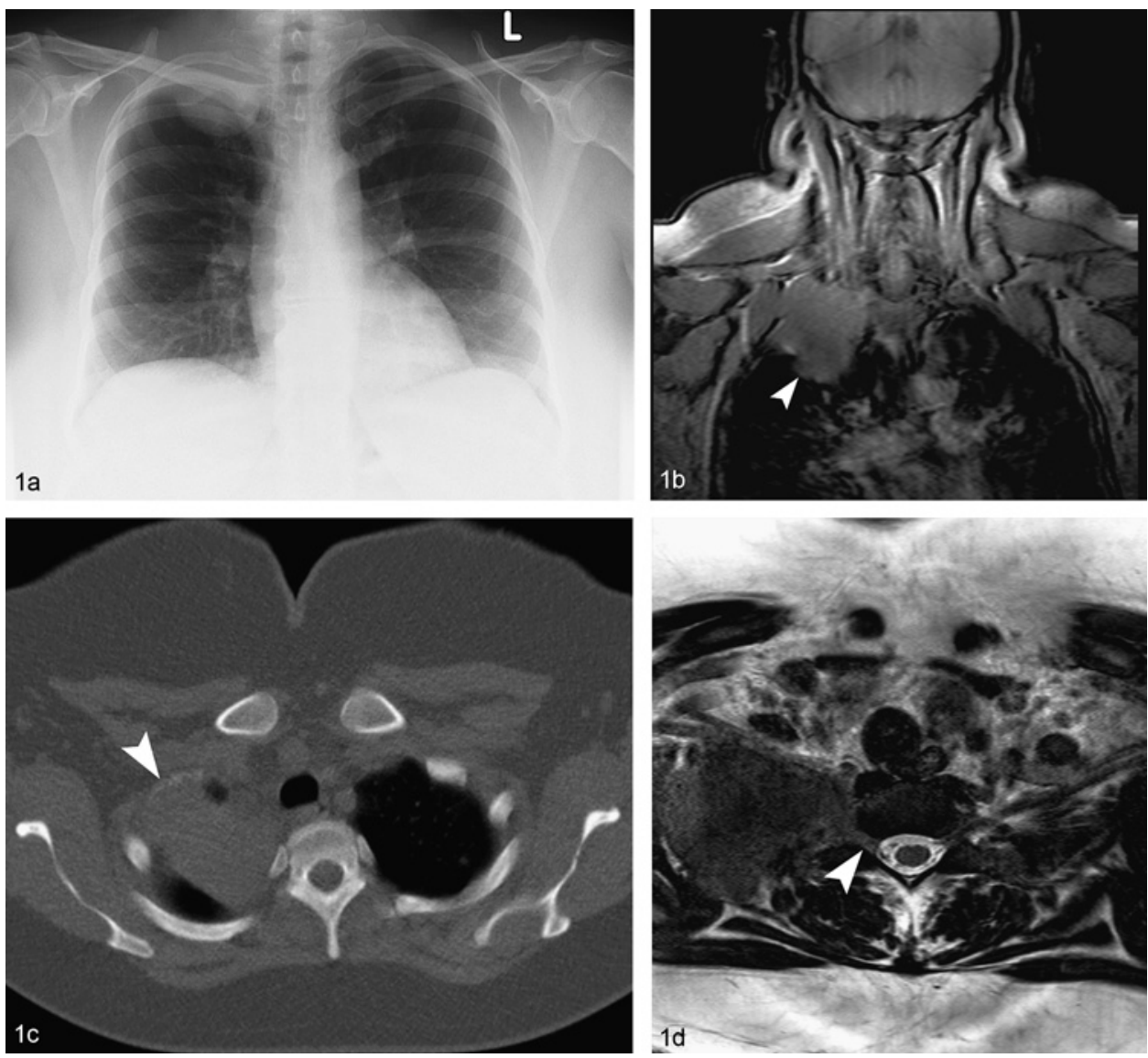

Figure 1 Conventional thorax images showing a mass in the right aperture measuring $4.5 \times 6 \times 6 \mathrm{~cm}(\mathrm{~A})$. MRI study of the brachial plexus showed a well-demarcated homogenous mass in the apex of the right hemithorax. The tumour was invading the thoracic wall, adjacent musculature and the right plexus brachialis and extended into the neuroforamen of C7-T1 (B, D). CT images showed evident destruction of the first rib on the right side (C).
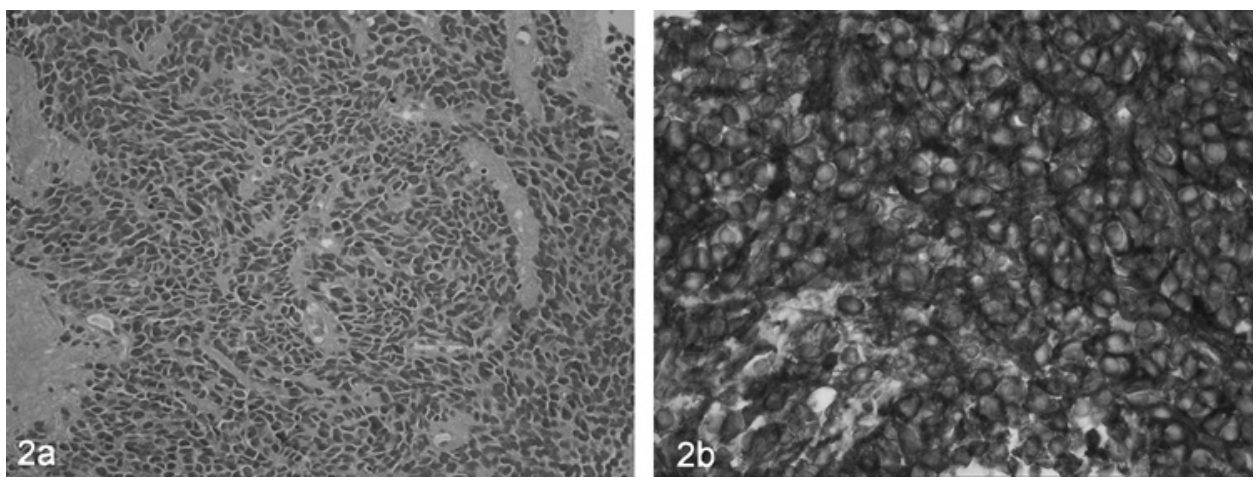

Figure 2 Histological examination of a CT-guided transthoracic needle biopsy showing a diffuse monotonous population of small round cells characterised by relatively little cytoplasm and round to ovoid hyperchromatic nuclei (A). A diffuse membranous staining for CD99 was present (B). 


\section{Learning points}

- Ewing's sarcoma of the first rib may cause Pancoast's syndrome.

- Most cases of Pancoast's syndrome are caused by lung cancer. However, the wide variety of diseases that can result in Pancoast's syndrome, including the present case, emphasises that a conclusive pathological diagnosis is mandatory. ${ }^{12}$

fluorescence in situ hybridisation, a diagnosis of an Ewing's sarcoma of the first rib was made.

\section{Lowie Vanfleteren, ${ }^{1}$ Rogier van Stiphout, ${ }^{2}$ Robert G Riedl, ${ }^{3}$} Rob Jansen, ${ }^{4}$ Dirk De Ruysscher, ${ }^{5,6}$ Anne-Marie C Dingemans ${ }^{1,6}$

${ }^{1}$ Department of Respiratory Medicine,Maastricht University Medical Center, Maastricht, The Netherlands; ${ }^{2}$ Department of Radiology, Maastricht University Medical Center, Maastricht, The Netherlands; ${ }^{3}$ Department of Pathology, Maastricht University Medical Center, Maastricht, The Netherlands; ${ }^{4}$ Department of Internal
Medicine, Maastricht University Medical Center, Maastricht, The Netherlands: ${ }^{5}$ Department of Radiotherapy, MAASTRO Clinic, Maastricht University Medical Center, Maastricht, The Netherlands; ${ }^{6} \mathrm{GROW}$ - School for Oncology and Developmental Biology, Maastricht University Medical Center, Maastricht, The Netherlands

Correspondence to Lowie Vanfleteren, Department of Respiratory Medicine, Maastricht University Medical Center, Postbus 5800, 6202 AZ Maastricht, The Netherlands; lowievanfleteren@gmail.com

Competing interests None.

Patient consent Obtained.

Provenance and peer review Not commissioned; externally peer reviewed.

Accepted 27 May 2010

Published Online First 7 September 2010

Thorax 2011;66:89-90. doi:10.1136/thx.2010.140004

\section{REFERENCES}

1. Arcasoy SM, Jett JR. Superior pulmonary sulcus tumors and Pancoast's syndrome. N Engl J Med 1997;337:1370-6.

2. Rusch VW. Management of Pancoast tumours. Lancet Oncol 2006;7:997-1005. 